\title{
Disruption of Brain White Matter Microstructure by Excessive Intracellular and Extracellular Fluid in Alcoholism: Evidence from Diffusion Tensor Imaging
}

\author{
Adolf Pfefferbaum $*, 1,2$ and Edith V Sullivan ${ }^{2}$ \\ 'Neuroscience Program, SRI International, Stanford University School of Medicine, USA; ${ }^{2}$ Department of Psychiatry and Behavioral Sciences, \\ Stanford University School of Medicine, USA
}

\begin{abstract}
Magnetic resonance diffusion tensor imaging (DTI) has revealed the disruption of brain white matter microstructure in normal aging and alcoholism undetectable with conventional structural MR imaging. The metrics of DTI can be useful in establishing the nature of the observed microstructural aberrations. Abnormally low fractional anisotropy (FA), a measure of diffusion orientation and coherence, may result from increased intracellular or extracellular fluid, which would be reflected in complementary high apparent diffusion coefficients (bulk mean diffusivity) and low FA, or from disorganization of fiber structure, which would be reflected in low FA but with a lack of the inverse FA and diffusivity relationship. To test these competing possibilities, we examined I 5 alcoholic men and $3 \mathrm{I}$ control men with DTI to quantify diffusivity in the genu and splenium of the corpus callosum and centrum semiovale. In addition to the previously observed FA deficits in all the three brain regions, the alcoholics had abnormally high white matter diffusivity values in the genu and centrum. Further, inverse correlations between FA and diffusivity were significant in the genu $(r=-0.52, p<0.05)$ and centrum $(r=-0.92, p=0.000 \mathrm{I})$. Multiple regression analyses examining diffusivity and age as predictors of FA identified diffusivity as a significant unique contributor to FA in both regions. These results suggest that decreased orientational coherence of brain white matter in alcoholism is attributable, at least in part, to the accumulation of intracellular and extracellular fluid in excess of that occurring in aging, and that the differential influence of these fluid compartments can vary across brain regions.
\end{abstract}

Neuropsychopharmacology (2005) 30, 423-432, advance online publication, 24 November 2004; doi: 10.1038/sj.npp. I 300623

Keywords: alcohol; diffusion; white matter; aging; anisotropy; trace; diffusivity

\section{INTRODUCTION}

White matter pathology is a consistent finding in the brains of alcohol-dependent individuals. Postmortem study of alcoholics has identified pathology in white matter's constituents and has noted demyelination (Lewohl et al, 2000; Tarnowska-Dziduszko et al, 1995), microtubule disruption (Paula-Barbosa and Tavares, 1985; Putzke et al, 1998), and axonal deletion, as well as morphological distortion involving cell processes (Harper et al, 1987; Pentney, 1991) and volume reduction arising from shrinkage or deletion of cell bodies, typical of Wallerian degeneration (Alling and Bostrom, 1980; Badsberg-Jensen and Pakkenberg, 1993; De la Monte, 1988; Harper and Kril,

\footnotetext{
*Correspondence: Dr A Pfefferbaum, Neuroscience Program, SRI International, 333 Ravenswood Avenue, Menlo Park, CA 94025, USA, Tel: + I 650859 2927, Fax: + I 650859 2743,

E-mail: dolf@synapse.sri.com

Received 6 July 2004; revised 21 September 2004; accepted 23 September 2004

Online publication: 20 October 2004 at http://www.acnp.org/citations/ Npp I02004040308/default.pdf
}

1991, 1993; Lancaster, 1993). Although postmortem study has been essential in identifying sources of microstructural and cytoskeletal abnormalities in alcoholism, the brain fixation process and postmortem collapse of fluid-filled spaces (eg, ventricles, sulci, and blood vessels) alter brain morphology from the living state; thus, postmortem results do not necessarily reflect the alcohol-related effects on the living brain (Pfefferbaum et al, 2004). Nonetheless, in vivo neuroimaging with conventional MRI has provided convergent validity for the gross white matter dysmorphology observed postmortem, by showing evidence for white matter volume shrinkage with alcoholic drinking (Agartz et al, 2003; Estruch et al, 1997; Hommer et al, 1996, 2001; Pfefferbaum et al, 1996, 1992; Symonds et al, 1999) and at least partial recovery of volume with abstinence (O'Neill et al, 2001; Pfefferbaum et al, 1995; Shear et al, 1994). Depiction of the gross anatomy of the living alcoholic brain was a critical initial step for verifying alcoholism-associated untoward effect on brain structure, but even high-resolution structural MRI has not enabled characterization of the microstructural integrity of the residual white matter volume in vivo. 
Recent developments in MR diffusion tensor imaging (DTI) afford a noninvasive approach for in vivo examination of the microstructure of brain tissue, particularly white matter (for reviews, see Basser and Jones, 2002; Kubicki et al, 2002; Lim and Helpern, 2002; Rosenbloom et al, 2003; Sullivan and Pfefferbaum, 2003). DTI permits assessment of water diffusion orientational coherence, measured as anisotropy, by quantifying the magnitude and orientation of water mobility on a voxel-by-voxel basis in a structure of interest. A tissue with high anisotropy is indicative of restricted diffusion typically in a regularly organized region, such as a white matter fiber. DTI studies in alcoholism are newly emerging and have revealed abnormally low anisotropy in white matter brain structures, in particular, the centrum semiovale and genu of the corpus callosum, implying disruption or disorganization of fiber structure. The alcohol-related deficits in white matter anisotropy exceed those observed in normal aging (Pfefferbaum et al, 2000b; Sullivan et al, 2001), cannot be accounted for by shrinkage in the underlying tissue mass (Pfefferbaum and Sullivan, 2003), relate to cognitive performance on tests of attention and working memory (Pfefferbaum et al, 2000a), and occur in both men (Pfefferbaum et al, 2000a) and women (Pfefferbaum and Sullivan, 2002).

Taken within the context of postmortem and ex vivo studies, compromised white matter fiber coherence detected in vivo with DTI may signify the breakdown of microstructural components marked by boggy tissue due to excessive fluid within or between cells or, alternatively, to cytoskeletal or extracellular collapse, for example, of myelin rather than from fluid invasion between myelin sheaths. To determine the contribution of excessive intracellular or extracellular fluid to low anisotropy in alcoholism, bulk mean diffusivity, another diffusion metric, needs to be invoked (c.f., Pierpaoli et al, 2001). Bulk mean diffusivity $(\langle D\rangle)$, derived from the trace of the tensor matrix, is the average magnitude of diffusion within a voxel and can be, but is not necessarily, inversely related to anisotropy (Pfefferbaum et al, 1999b; Pierpaoli et al, 2001). When high diffusivity accompanies low anisotropy, the imaged tissue is characterized by poor coherence and excess fluid, as occurs in regions of tissue recovery following resolution of ischemic stroke (Moseley et al, 1990; Rumpel et al, 1998). Alternatively, when diffusivity and anisotropy are poorly related and are both low, as occurs in the early stages of stroke (Bykowski et al, 2004; Pierpaoli et al, 2001), disrupted intravoxel coherence is characterized by disordered cellular microstructure, despite cell swelling, which increases diffusivity (Sehy et al, 2002). Nonetheless, intracellular fluid accounts for about $85 \%$ of brain tissue water and dominates diffusivity in normal and ischemic tissue (Silva et al, 2002).

Given that DTI enables in vivo quantitative characterization of tissue anisotropy and diffusivity on a microstructural (that is, intravoxel) basis, the current analysis tested two competing hypotheses to determine the nature of low anisotropy in alcoholics: (1) a complementary relationship between low anisotropy and high diffusivity would support excessive fluid as contributing to fiber structure disorganization; (2) lack of regional low anisotropy/high diffusivity correlations would indicate an alternative cause of intravoxel incoherence, possibly implicating breakdown of cytoskeletal constituents, such as microtubules (Ringer et al, 2001), without concomitant increase in intracellular or interstitial fluid. To test these hypotheses, we quantified trace from subjects in our previously published DTI study of regional white matter anisotropy in alcoholic and control men (Pfefferbaum et al, 2000a). The regions selected, the genu and splenium of the corpus callosum and centrum semiovale, are two large regions of supratentorial white matter known to show volume shrinkage on in vivo MRI and neuropathological abnormalities on postmortem examination in alcoholism. Neuropathology in these regions may set the stage for development of serious alcoholism-related white matter disorders, such as Marchiafava-Bignami disease or central pontine myelinolysis. Here, we examined group differences in regional diffusivity, measured the relationship between anisotropy and diffusivity, and determined the variance in anisotropy accounted by the independent contributions of age, alcohol consumption, and diffusivity. To extend the characterization of the water diffusion in each white matter region, we parsed the diffusion ellipsoid into three geometric componentslinear, planar, and spherical - each describing a predominant shape of the diffusion ellipsoid, defined and limited by the local structure (Alexander et al, 2000; Pfefferbaum and Sullivan, 2003). We tested the hypothesis that the degree of anisotropy of the alcoholics would best be predicted by a spherical (indicative of high fluid presence) rather than a linear (indicative of highly regular structure) model of water molecular diffusion. Finally, in a follow-up to our previous observation of a selective relationship between splenium anisotropy and working memory test performance in alcoholics, we tested whether working memory was better predicted by anisotropy, an index of fiber integrity and coherence, or by diffusivity, an index of excessive fluid presence in white matter.

\section{PATIENTS AND METHODS}

\section{Subjects}

The alcoholic group (Table 1) comprised 15 men (40-72 years old), recruited from local inpatient and outpatient treatment facilities to participate in our brain-imaging studies. Potential participants were interviewed with the SCID and received a DSM-IV diagnosis of substance dependence for alcohol. Screening excluded patients with a history of hospitalization for DSM-III-R or DSM-IV diagnosis of schizophrenia, major affective disorders, or medical or neurological conditions affecting CNS, seizure disorder unrelated to alcohol withdrawal, diphenylhydantoin or steroid use in the past month, or the meeting criteria for substance abuse other than alcohol within the past year. One alcoholic man met the DSM-IV criteria for substanceinduced persisting amnestic disorder (that is, alcoholic Korsakoff's syndrome), and had a difference of 40 points between his estimated premorbid IQ, as assessed by the national adult reading test (NART) (Nelson, 1982), and the General Memory Index of the Wechsler Memory Scalerevised (Wechsler, 1987). Subjects also underwent physical examinations and a semi-structured alcohol consumption assessment interview (Pfefferbaum et al, 1992; Skinner, 1982; Skinner and Sheu, 1982). The median time between 
Table I Subject Group Characteristics (Means and SD)

\begin{tabular}{|c|c|c|c|c|c|c|}
\hline Group & Age & Education & NART IQ & WMS-R GMI & DRS total & Lifetime alcohol consumption (kg) \\
\hline Control & 54.4 & 16.1 & 114.9 & 109.4 & $\mid 40.1$ & 65.9 \\
\hline$[N=15]$ & {$[12.3]$} & {$[2.6]$} & {$[6.5]$} & {$[19.2]$} & {$[4.0]$} & [99.9] \\
\hline Alcoholic & 55.4 & 14.5 & 109.5 & 104.5 & |36.| & 1484.6 \\
\hline$[N=19]$ & {$[9.6]$} & {$[2.6]$} & {$[7.9]$} & {$[16.3]$} & {$[6.2]$} & {$[1 \mid 93.7]$} \\
\hline
\end{tabular}

For controls, $N=9$ for WMS-R GMI, $N=16$ for DRS total, $N=17$ for alcohol consumption.

the last drink and MRI scanning was 332 days (range $=25-$ 5110 days).

The healthy control subjects (Table 1) were 31 men (2376 years old), recruited from the local community, and formed the group on which adjustment for normal variation in age was calculated (Pfefferbaum et al, 1994). Of this group, 19 men (40-76 years old) were matched in age to the alcoholic patients and were used in control $v s$ alcoholic group comparisons. All subjects completed a detailed questionnaire inquiring about current and past medical and psychiatric conditions, medications, and substance use; 13 subjects were also given the SCID and a physical examination. Demographic and alcohol history information for the alcoholic and control groups is presented in Table 1.

\section{MRI Acquisition Protocols}

All scans were acquired on a $1.5 \mathrm{~T}$ GE Signa with Horizon EchoSpeed gradients. A fuller description can be found in Pfefferbaum et al (2000b).

Anatomical MRI. On the midsagittal image from a sagittal, spin-echo scout sequence, the anterior and posterior commissures (AC-PC) were identified. For tissue segmentation, a fast spin-echo (FSE) sequence was collected $(5 \mathrm{~mm}$ thick slices, $0 \mathrm{~mm}$ slice gaps, $\mathrm{TR}=2500 \mathrm{~ms}, \mathrm{TE}=20 / 80 \mathrm{~ms}$, echo train length $=8,256 \times 256$ acquisition matrix, field of view $=24 \mathrm{~cm}, 18$ slices beginning $2 \mathrm{~cm}$ below and aligned with the AC-PC).

Diffusion tensor imaging (DTI). DTI was performed using a single-shot spin-echo echo-planar imaging technique $(5 \mathrm{~mm}$ thick slices, $0 \mathrm{~mm}$ slice gaps, $\mathrm{TR}=6000 \mathrm{~ms}$, $\mathrm{TE}=106 \mathrm{~ms}, 128 \times 128$ zerofilled to a $256 \times 256$ acquisition matrix, field of view $=24 \mathrm{~cm}, 18$ slices). The slice locations were identical to the anatomical MRI. The amplitude of the diffusion-sensitizing gradients was $1.4 \mathrm{Gauss} / \mathrm{cm}$, with $32 \mathrm{~ms}$ duration and $34 \mathrm{~ms}$ separation. This resulted in a total $b$-value of $860 \mathrm{~s} / \mathrm{mm}^{2}$.

Diffusion was measured along six noncollinear directions: $(x, y, z)=[(1,1,0),(0,1,1),(1,0,1),(-1,1,0),(0,-1,1)$, $(1,0,-1)]$. For each gradient direction, four images were acquired and averaged. Two images with no diffusion weighting $\left(b=0 \mathrm{~s} / \mathrm{mm}^{2}\right)$ were acquired and averaged, and two similar images $\left(b=0 \mathrm{~s} / \mathrm{mm}^{2}\right)$ with a CSF-attenuating inversion recovery pulse were also acquired and averaged.
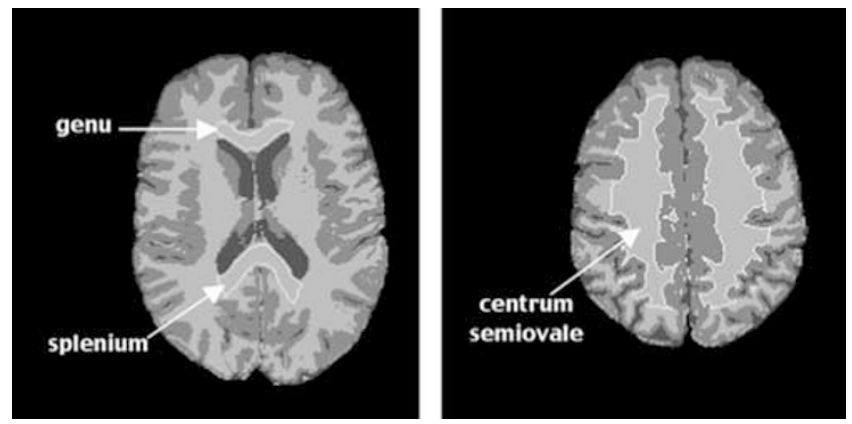

Figure I Segmented axial MR images with geometric identification of regions of interest for application to FA and mean diffusivity images.

\section{Image Processing}

MRI tissue segmentation. Tissue segmentation was performed with a modified version (Pfefferbaum et al, 1999a) of the automated three-compartment model of Lim and Pfefferbaum (1989), which took advantage of the different tissue contrast in short- and long-echo spin-echo data. The contents of the intracranial cavity (brain and cerebrospinal fluid (CSF)) were separated from the skull and external tissue with a stripping algorithm that used the bright peripheral CSF signal to define a margin. The images were then filtered to remove radiofrequency inhomogeneities with a homomorphic spatial filter. Long-echo images were subtracted from short-echo images to enhance the CSF/ tissue contrast, and the image was segmented into CSF and tissue (Kittler and Illingworth, 1986). The CSF was then used as a mask on the short-echo image, and a nonparametric histogram operator (Otsu, 1979) was applied to separate gray matter from white matter (Figure 1). All analyses were performed blind to subject identity.

Visually identified segmentation errors in white matter regions, primarily around the posterior horns of the lateral ventricles, classified as CSF or gray matter were manually edited into the white matter compartment. An automated program was used to classify pixels as WMHIs by their proton density and T2 characteristics. Two-point T2 and proton density (ie, the intercept from the T2 computation approximating proton density) images were constructed. A nonparametric histogram operator roughly bisected the proton density distribution and a histogram of the T2 values in the pixels with high proton density was constructed. Pixels in the edited white matter compartment with T2 
values greater than $2 \mathrm{SD}$ from the mean were classified as WMHIs. In the control group, WMHI volumes in each region of interest correlated significantly with age (genu $r=0.64$, splenium $r=0.56$, centrum $r=0.71, p \leqslant 0.001$ ). When correcting the regional WMHI for the influence of normal age, the alcoholics did not differ significantly from the controls in WMHI volume in any region ( $t$-tests for alcoholics $>$ controls, $p>0.78$ for all regions). On average, WMHIs accounted for only $2-4 \%$ of the total regional volumes. WMHIs were not excluded from any region of interest because WMHIs are a normal variant of aging (eg, Jernigan et al, 1991; this report).

Regions of interest. For DTI analysis, the regions of interest were determined from the native anatomical MR images, and the DTI data were classified as white matter, gray matter, or CSF based on the segmentation map, not the FA maps, thus avoiding the problem of using the dependent variable (ie, FA) to define the anatomic regions. The white matter regions selected for study were those best viewed in the axial plane. The genu and splenium of the corpus callosum were indexed separately and measured on the three successive slices on which they were fully volumed. The anterior and posterior margins of the genu and splenium were identified with a combination of manual and automated region of interest drawing and segmentation overlay. The lateral extents of these two structures were determined with a morphological erosion operator used to define the medial $30 \%$ of the slice. The region representing the centrum semiovale was composed of white matter in the medial $55 \%$ of the left and right hemispheres taken from three contiguous slices, starting from the most superior slice that included a fully volumed lateral ventricle in at least one hemisphere (Figure 1).

\section{DTI Quantification}

As described previously (Pfefferbaum et al, 2000a, b), DTI quantification was preceded by eddy current correction based on the inversion recovery images, which were used to unwarp eddy current-induced image distortions that vary from one diffusion direction to the next in the diffusionweighted images (de Crespigny and Moseley, 1998). This procedure employed a six-parameter affine correction on a slice-by-slice basis (Woods et al, 1998).

Using the eddy current-corrected averaged images with $b=0$ and $860 \mathrm{~s} / \mathrm{mm}^{2}$, six maps of the apparent diffusion coefficient (ADC) were calculated, each being a sum of two diagonal elements and one off-diagonal element of the diffusion tensor. Solving the six independent equations with respect to ADCxx, ADCxy, etc yielded the elements of the diffusion tensor. The trace of the tensor matrix (the sum of the eigenvalues) and bulk mean diffusivity (the mean of the eigenvalues), like FA, were calculated on a voxel-by-voxel basis.

The general diffusion tensor was then diagonalized, yielding eigenvalues $\lambda_{1}, \lambda_{2}, \lambda_{3}$, as well as eigenvectors that define the predominant diffusion orientation. Based on the eigenvalues, the fractional anisotropy (FA) was calculated on a voxel-by-voxel basis. Thus, each diffusion-weighted study was reduced to a set of three images for each slice
(FA, diffusivity, and $b=0$ ) to be used for analysis in conjunction with the anatomical images.

Ellipsoid shape analysis. As described previously (Pfefferbaum and Sullivan, 2003), FA is a standardized measure reflecting the degree to which one of the three lambdas calculated from the tensor matrix dominates the other two. To take advantage of quantitative information about the separate contributions of the nondominant lambdas and their relationship to each other, we characterized the shape of the diffusion ellipsoid following the analysis approach of Alexander et al (2000). This approach models the shape of the diffusion ellipsoid as a combination of linear, planar, and spherical shapes (Westin et al, 2002).

Spatial registration DTI protocol. In order to accurately superimpose the structural and FA images, we derived a warping function to correct the spatial distortion inherent in echo-planar imaging. This procedure (third-order, twodimensional polynomial function; Woods et al, 1998, 1992)) was used to optimize the fit of the $b=0$ and late-echo FSE (that is, anatomical MRI) images and then applied to the diffusion-weighted images. This approach avoided using the dependent variable in the warping procedure. Although this procedure can be applied before or after tensor calculation, warping the basis images prior to FA and diffusivity computation was desirable, as it allowed the generation of spatially warped eigenvector data for the computation of intervoxel coherence (C; not used in this report), which can be used to examine voxel-to-voxel orientational coherence (Pfefferbaum and Sullivan, 2002; Pfefferbaum et al, 2000a) and avoid the problem of phase wraparound when applying warping functions to eigenvector data (Pfefferbaum et al, 2000b).

\section{Working Memory Testing}

The alcoholic men received three tests of working memory: Backward Digit Span, Backward Block Span, and Trail Making Part B. Following our previously published method (Sullivan et al, 1994), single working memory composite score was derived by transforming the individual test scores to age-corrected standardized $Z$-scores based on control values and then taking the mean of the three individual test $Z$-scores for each alcoholic man. Scores below 0 are in the direction of impairment.

\section{Statistical Analysis}

Group differences in diffusivity were examined with repeated-measures analysis of variance (ANOVA) and follow-up unpaired $t$-tests between the group of 15 alcoholic patients and the age-matched group of 19 controls. Analyses were conducted twice: once on values adjusted with regression analysis based on values from the 31 control men to remove the observed effects of normal aging and to express group results in terms of effect size, and then again on the median values without age adjustment to determine the variation in magnitude of diffusivity across regions (Pfefferbaum et al, 2000b). The resulting values were standardized age-corrected $Z$-scores, where the expected mean of the controls was $0 \pm 1$ SD (Pfefferbaum et al, 1992). 
Correlations between DTI measures and demographic and alcohol variables were performed with Pearson tests. All comparisons were tested two-tailed (alpha $=0.05)$.

\section{RESULTS}

Regional mean FA values and correlations with demographic and alcohol variables of these groups were reported previously (Pfefferbaum et al, 2000a) and do not appear herein. For a visual display of group differences, Figure 2 presents the average images of FA and diffusivity for the 15 alcoholics and 19 controls. Grand average images were constructed for illustrative purposes (Figure 2). Each individual's slices were spatially normalized to allow structural alignment. The segmented structural images were aligned to those of an index control chosen at random using
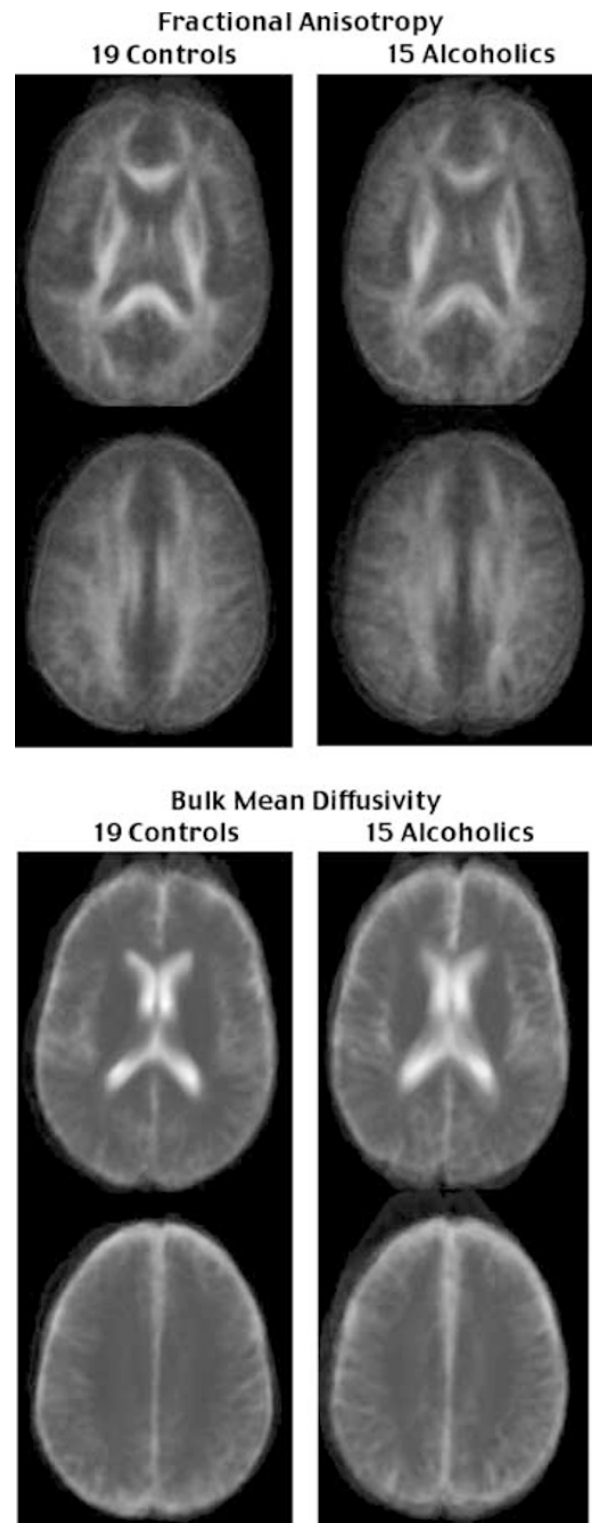

Figure 2 Averaged images at two levels in the brains of the 15 alcoholics and 19 controls for FA (top panel of four images) and mean diffusivity (bottom panel of four images). a two-dimensional, six-parameter, affine model (AIR) (Woods et al, 1998). The transformation for each subject was then applied to his FA and diffusivity images, and a grand average constructed by simple averaging on a pixelby-pixel basis.

\section{Group Differences in Mean Diffusivity}

For age-corrected $Z$-scores of diffusivity, a two-group $\times$ three-region ANOVA yielded a significant group effect $(\mathrm{F}(1,32)=4.603, p=0.0396)$, but neither a region effect $(\mathrm{F}(2,64)=1.473$, NS) nor interaction $(\mathrm{F}(2,64)=2.101, \mathrm{NS})$. Relative to the controls, the alcoholics had greater diffusivity values in all the three regions; the difference was significant in the centrum $(t(32)=2.696, p=0.0111)$ and showed a trend in the genu $(t(32)=2.022, p=0.0517)$.

The raw-scores ANOVA yielded a trend for a group effect $(\mathrm{F}(1,32)=3.806, p=0.0599)$, a significant region effect $(\mathrm{F}(2,64)=174.294, \quad p=0.0001), \quad$ and no interaction $(\mathrm{F}(2,64)=2.149, p=0.125)$. The pattern of group differences for the raw mean diffusivity values of each region followed that of the $Z$-scores (Figure 3 ), where diffusivity was significant in the centrum $(t(32)=2.433, p=0.0207)$, showed a trend in the genu $(t(32)=1.876, p=0.0698)$, and
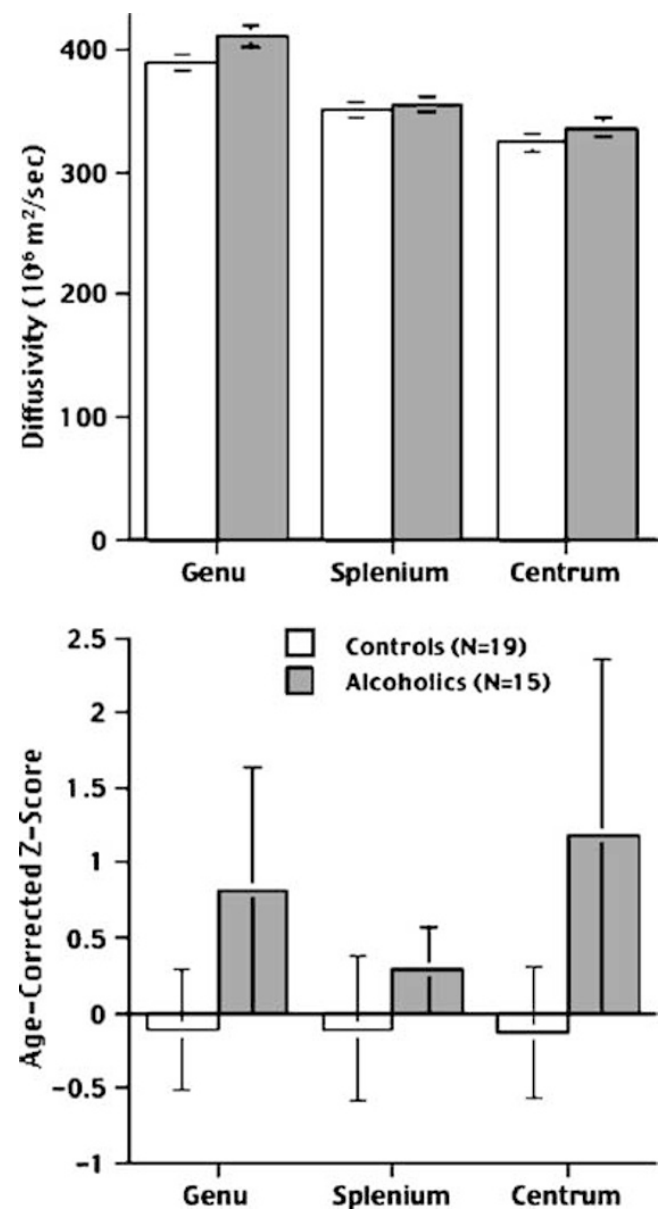

Figure 3 Raw values and age-corrected Z-scores ( \pm SEM bars) of regional callosal and centrum trace. Alcoholics had higher mean diffusivity than controls despite equivalent tissue volumes of the regional samples. The alcohol effect was most profound in the centrum ( $>$ ISD). 

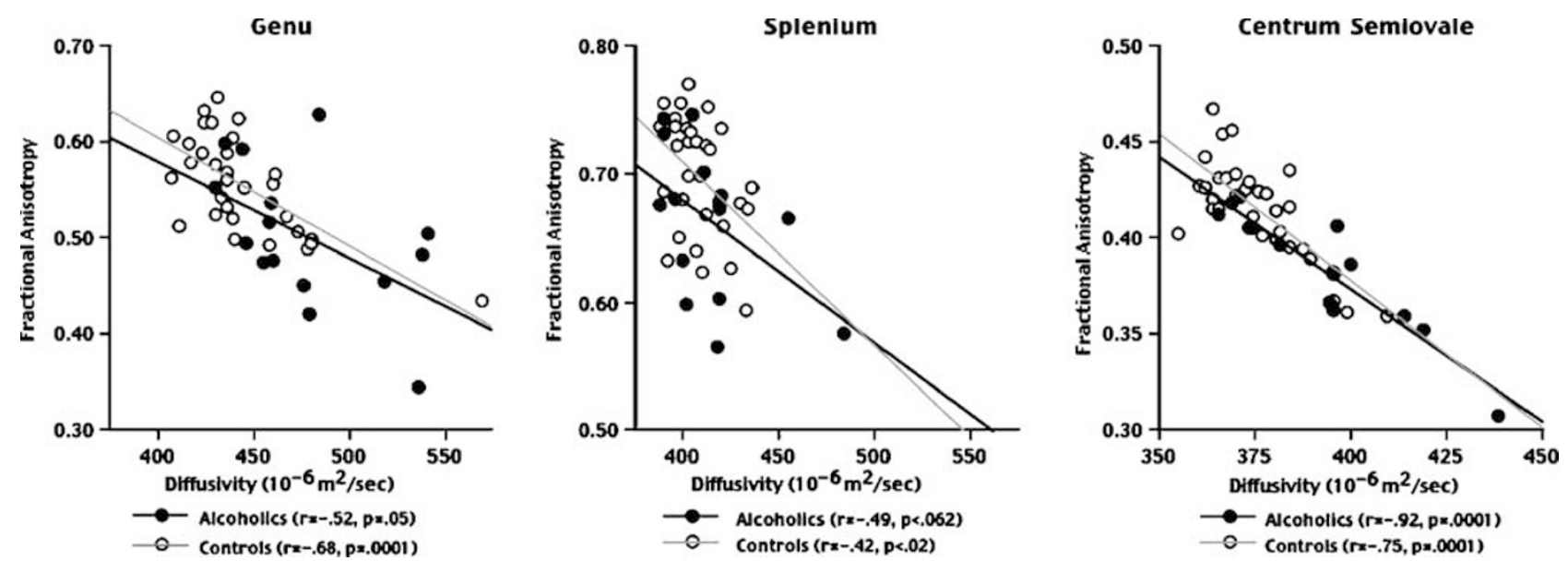

Figure 4 Lower FA and higher mean diffusivity were related to each other in controls and alcoholics.

no effect in the splenium $(t(32)=0.4448$, NS). Trace group effects could not be attributed to group differences in the tissue volume on which the DTI measures were based because there were none (genu: $t(32)=1.445, p=0.1582$; splenium $t(32)=1.061, p=0.2967$; centrum $t(32)=0.500$, $p=0.6208)$.

\section{Correlates of Mean Diffusivity}

Correlations across subjects within each group involving diffusivity were based on raw values (ie, non-age-corrected values). In all three regions, diffusivity correlated inversely with FA in both controls and alcoholics (Figure 4). The correlations between diffusivity and age in the alcoholics were significant in the centrum, modest in the splenium, and not significant in the genu. Total lifetime consumption of alcohol did not correlate with diffusivity in any region; lack of observable relationship may be related to the wide range in length of sobriety in the alcoholics and reflect variability in point of recovery. The diffusivity-age correlation pattern in the controls differed from the alcoholics with respect to the genu; specifically, the controls showed significant correlations between age and diffusivity in the genu and centrum, and a nonsignificant trend in the splenium (Table 2 ).

We next tested the value of diffusivity and age in predicting FA. Together, diffusivity and age accounted for $39 \%$ of the FA variance in the genu, $27 \%$ in the splenium, and $87 \%$ in the centrum in the alcoholics. To test the independent contributions of age and diffusivity to FA in each group, a series of multiple regression analyses entered regional diffusivity and age as simultaneous predictors of the associated regional FA. In general, these analyses indicated that diffusivity made a greater independent contribution to FA than did age; however, the predictive value of diffusivity over age was significant only in the centrum (Table 3). The same analyses applied to the controls yielded a pattern different from that of the alcoholics in the genu and centrum. Specifically, diffusivity and age together accounted for $60 \%$ of the variance in the genu, $28 \%$ in the splenium, and $75 \%$ in the centrum. Multiple regression analysis using age and diffusivity as
Table 2 Correlations with Age (Raw Values)

\begin{tabular}{|c|c|c|c|c|}
\hline & \multicolumn{2}{|c|}{ Alcoholics $(n=15)$} & \multicolumn{2}{|c|}{ Controls $(n=19)$} \\
\hline & $r$ & $p$ & $r$ & $p$ \\
\hline \multicolumn{5}{|l|}{ Diffusivity } \\
\hline Genu & 0.26 & NS & 0.66 & 0.002 \\
\hline Splenium & 0.42 & NS & 0.38 & 0.110 \\
\hline Centrum & 0.63 & 0.012 & 0.57 & 0.011 \\
\hline \multicolumn{5}{|l|}{ FA components } \\
\hline \multicolumn{5}{|l|}{ Genu } \\
\hline Linear & -0.41 & NS & -0.56 & 0.012 \\
\hline Planar & -0.61 & 0.016 & -0.18 & NS \\
\hline Spherical & 0.55 & 0.033 & 0.58 & 0.009 \\
\hline \multicolumn{5}{|l|}{ Splenium } \\
\hline Linear & -0.40 & NS & -0.45 & 0.053 \\
\hline Planar & -0.13 & NS & -0.05 & NS \\
\hline Spherical & 0.35 & NS & 0.39 & 0.100 \\
\hline \multicolumn{5}{|l|}{ Centrum } \\
\hline Linear & -0.68 & 0.006 & -0.72 & 0.001 \\
\hline Planar & -0.56 & 0.030 & -0.67 & 0.002 \\
\hline Spherical & 0.64 & 0.011 & 0.72 & 0.001 \\
\hline
\end{tabular}

predictors of FA in the controls revealed significant independent contribution from diffusivity to genu FA and from diffusivity and age to centrum FA (Table 3 ). The total lifetime consumption of alcohol did not correlate with diffusivity in any region in the alcoholics.

\section{Ellipsoid Shape Analysis}

The first set of correlations tested the relationship between the three shape dimensions and age. We predicted that the 
Table 3 Multiple Regression Analysis: Predictors of FA (Raw Values)

\begin{tabular}{|c|c|c|c|c|c|}
\hline \multirow[b]{2}{*}{ Predictor } & \multirow[b]{2}{*}{ Predicted } & \multicolumn{2}{|c|}{ Alcoholics $(N=15)$} & \multicolumn{2}{|c|}{ Controls $(N=19)$} \\
\hline & & F (partial) & $p$ & F (partial) & $p$ \\
\hline & Genu FA & & & & \\
\hline Genu diffusivity & & 3.33 & 0.100 & 9.72 & 0.007 \\
\hline Age & & 2.40 & 0.150 & 0.70 & 0.410 \\
\hline & Splenium FA & & & & \\
\hline Splenium diffusivity & & 2.37 & 0.150 & 2.59 & 0.130 \\
\hline Age & & 0.45 & 0.510 & 1.40 & 0.260 \\
\hline & Centrum FA & & & & \\
\hline Centrum diffusivity & & 37.86 & 0.000 & 15.17 & 0.002 \\
\hline Age & & 0.98 & 0.340 & 5.99 & 0.030 \\
\hline
\end{tabular}

Table 4 Multiple Regression Analysis: Alcoholics $(N=15)$ (Raw Values)

\begin{tabular}{|c|c|c|c|c|}
\hline \multirow[b]{3}{*}{ Predictors } & \multicolumn{4}{|c|}{ Predicted } \\
\hline & \multicolumn{2}{|c|}{ FA } & \multicolumn{2}{|c|}{ Diffusivity } \\
\hline & F (partial) & $p$ & F (partial) & $p$ \\
\hline \multicolumn{5}{|l|}{ Genu } \\
\hline Linear & 43.04 & 0.000 & 0.66 & 0.436 \\
\hline Planar & 0.04 & 0.846 & 5.36 & 0.041 \\
\hline Spherical & 10.46 & 0.008 & 2.33 & 0.156 \\
\hline \multicolumn{5}{|l|}{ Splenium } \\
\hline Linear & 10.15 & 0.008 & 0.26 & 0.623 \\
\hline Planar & 0.08 & 0.788 & 0.01 & 0.918 \\
\hline Spherical & 6.88 & 0.024 & 0.58 & 0.464 \\
\hline \multicolumn{5}{|l|}{ Centrum } \\
\hline Linear & $|5.8|$ & 0.002 & 0.49 & 0.498 \\
\hline Planar & 3.23 & 0.100 & 2.70 & 0.129 \\
\hline Spherical & 36.61 & 0.000 & 0.01 & 0.924 \\
\hline
\end{tabular}

greater the degree to which the diffusion could be described as linear or planar, the younger the individual, whereas diffusion described by a spherical ellipsoid would be associated with older age. In general, this pattern was present in both the alcoholics and controls, although none of the correlations was significant in the splenium (Table 2).

We then examined the independent predictive value of the three shape dimensions to FA in the alcoholics. For the genu, splenium, and centrum, the linear and the spherical dimensions were significant independent predictors of FA in the respective regions. When these analyses were applied to the mean diffusivity, only one variable emerged as a significant independent predictor: the planar component predicted genu diffusivity, but the effect was modest $(p<0.05)$ (Table 4).

\section{Predictors of Working Memory}

Previously, we reported that working memory performance by these alcoholic subjects showed a selective relationship with FA $Z$-scores (ie, age-corrected values) in the splenium (Pfefferbaum et al, 2000a). Here we questioned whether this relationship could be attributed to FA (a marker of fiber coherence) or to diffusivity (an index of intracellular or extracellular fluid content). Multiple regression analysis, based on $Z$-scores for FA, diffusivity, and working memory, identified $\mathrm{FA}$ as a unique predictor of working memory (partial $\mathrm{F}=8.465, p=0.0131$ ) over and above the contribution from diffusivity (partial $\mathrm{F}=1.278, p=0.2804$ ).

\section{DISCUSSION}

The aim of this in vivo MR diffusion imaging analysis was to test specific hypotheses regarding the presence and relevance of excessive intracellular and extracellular fluids in explicating the nature of microstructural degradation in selected regions of white matter of the brains of alcoholics. The structures targeted were the genu and splenium of the corpus callosum and centrum semiovale. It had already been established that these alcoholic men had significant disruption of brain white matter microstructure, observed as low regional anisotropy, beyond that occurring in normal aging. The present analysis addressed whether the abnormally low anisotropy, an index of fiber orientational coherence on the intravoxel level, resulted from increased fluid, which would be reflected in complementary high diffusivity, or from disorganization of fiber structure, which would be reflected in low anisotropy but with a lack of the complementary inverse correlation with diffusivity. We observed that, in addition to the abnormally low anisotropy in all the three regions examined, the alcoholics exhibited abnormally high diffusivity in the genu and centrum. Further, low anisotropy was related to high diffusivity in all the three regions, with the highest inverse correlation in the centrum and the lowest in the splenium. Analyses examining diffusivity and age as predictors of anisotropy identified diffusivity over age as a significant unique contributor to anisotropy in the centrum and genu. These results suggest that decreased brain white matter intravoxel coherence in alcoholism is attributable, at least in part, to the accumulation of interstitial or intracellular fluid or both fluid compartments, especially in the centrum, in excess of that occurring with advancing age.

It is not directly discernable from DTI data whether the higher diffusion in the white matter of alcoholics receives a greater contribution from excessive fluid in intracellular or in extracellular space. A common assumption is that high diffusion is associated with fluid in extracellular space, where water motility is less obstructed than in intracellular space, which is crowded by cytoskeleton (Norris et al, 1994; Rumpel et al, 1998). A recent report based on rodent models of stroke challenges this assumption (Silva et al, 2002). Silva et al estimated that $85 \%$ of the total apparent diffusion coefficient (ADC), an index of diffusivity, could be attributed to intracellular fluid in a sample of rat brain tissue. Using an isolated Xenopus oocyte model, Sehy et al (2002) demonstrated that an increase in intracellular fluid produced increases in both the slow and fast components of 
$\mathrm{ADC}$, calling into question the use of fast $v$ s slow ADC as a marker distinguishing intracellular from extracellular fluid. It is unlikely that the chronic alcoholics in our study had acute cell swelling as seen in early stroke. Converging evidence from molecular studies of microtubule integrity in rodent models of alcoholism and ischemic stroke suggests that the mainstay of the high diffusivity in the current study could arise from disruption of cell cytoskeleton. In particular, a molecular analysis of the brains of alcoholpreferring AA rats chronically exposed to high doses of alcohol revealed substantially lower levels of microtubuleassociated protein-2 (MAP-2) mRNA in selective brain regions than in nonexposed rats, indicating disruption of cytoskeletal integrity by alcohol (Putzke et al, 1998). The cerebral ischemia study examined whether the high diffusion, measured with MR diffusion-weighted imaging in the hours immediately following stroke produced by occlusion, was related to MAP-2 or to the astrocytic marker, glial fibrillary acidic protein (GFAP) immunoreactivity. The results revealed significant loss of MAP-2 immunoreactivity in the ischemic area with high diffusion, indicative of microtubule compromise, but not of GFAP, indicative of morphologically normal astrocytes (Ringer et al, 2001). All of these factors, taken together within the context of the regionally different relationships among FA, diffusivity, and age observed in the alcoholics in the present study, lead to the following speculations: Overall, intravoxel diffusivity is greater in the alcoholics than controls and arises primarily from the intracellular compartment, regardless of the degree of relationship between FA and diffusivity. In cases where FA and diffusivity show strong negative correlations, such as the centrum semiovale and to a less extent the genu, fluid presence in the extracellular compartment is also contributory to local diffusivity. Where FA and diffusivity are less strongly correlated, such as the splenium, the intracellular compartment may predominate.

Constituents of white matter are vulnerable to alcoholism's untoward effects from multiple sources. Nutritional deficiency, a common concomitant of chronic alcoholism, also underlies several serious conditions involving white matter deterioration. Exemplary conditions (Victor et al, 1989) include central pontine myelinolysis and Marchiafava-Bignami disease. The former is marked by focal demyelination of the pons basalis, caused by excessively rapid correction of hyponatremia and possibly folate depletion, resulting in quadriplegia in severe cases. Marchiafava-Bignami disease is marked by severe demyelination, classically, of the corpus callosum and anterior commissure, extending in some cases laterally to the centrum semiovale and inferiorly to the optic nerve, tract, and chiasm, and is related to thiamine deficiency (Gass et al, 1998) and generally poor nutrition (Rickert et al, 2001), resulting in mild to severe cases of cerebral hemisphere disconnection syndrome. One study demonstrated that even a single bout of thiamine deficiency can cause significant cortical and subcortical degeneration of white matter axons (Langlais and Zhang, 1997). Perhaps the most basic mechanism of alcohol-induced brain tissue disruption is from acetaldehyde, alcohol's primary metabolite and mediator of microtubule system impairment (Pratt et al, 1990). It has been speculated that acetaldehyde interacts with nutritional deficiencies to exacerbate its untoward outcome, as evidenced from the graver clinical state rendered by these nutritional deficiencies in alcoholics than in nonalcoholics (Pratt et al, 1990). Further, alcoholism influences genetic regulation of myelin production and repair by downregulating myelin-related genes (Lewohl et al, 2000).

Chronic, uncomplicated alcoholism is not marked by space-occupying lesions, but does result in brain tissue volume shrinkage, expansion of ventricular and sulcal spaces, and disruption of the brain's microstructure. Thus, rather than a 'complete' lesion resulting in focal disconnection syndromes, alcoholism appears to be characterized by 'incomplete' lesions, in the form of degradation of cell microstructure, that are likely contributors to the mild, yet consistently observed, cognitive and motor deficits in alcoholism (for reviews, see Fein et al, 1990; Nixon, 1993; Oscar-Berman, 2000; Sullivan, 2000). In particular, alcoholism-related disruption of white matter fibers of the genu may contribute to frontal lobe pathology reported in vivo and postmortem and to neuropsychological deficits of executive functions, including processing inefficiency (De Rosa et al, 2004; Nixon et al, 1995) and problem solving (Moselhy et al, 2001; Oscar-Berman, 2000; Sullivan, 2000), typical of chronic alcoholism. Further, disruption of callosal and centrum fiber tracts may serve to limit the flexibility in pathways recruited to execute complex cognitive and motor tasks requiring the coordination of widely distributed networks. Some of these speculations have received support from our earlier DTI study of uncomplicated alcoholism, which reported a double dissociation of brain structurefunction relationships: measures of attention but not working memory correlated with intervoxel anisotropy in the genu but not the splenium, whereas measures of working memory but not attention correlated with intravoxel anisotropy in the splenium but not the genu (Pfefferbaum et al, 2000a).

In vivo MRI studies tracking the course of alcoholism report shrinkage of brain white matter with active drinking and a trend toward volume normalization with abstinence (Pfefferbaum et al, 1995; Shear et al, 1994). White matter constituents and architecture, including cytoskeleton, microtubules, oligodendrocytes, and cell body extensions, which are capable of remodeling following injury, are candidate contributors to such tissue recovery. Given the success of DTI in predicting tissue viability following stroke, the combined DTI measures of diffusion and anisotropy provide a noninvasive, quantitative approach for a longitudinal study of alcoholics in predicting brain regions and tissue likely to have successful recovery and restitution of function. These possibilities underscore the value of examining additional white matter systems highly vulnerable to chronic alcoholism, such as the pontocerebellar tracts and beyond (Sullivan, 2003), in alcoholic men and women, both while actively drinking and during the course of recovery.

\section{ACKNOWLEDGEMENTS}

A partial report of these data was presented at the Research Society on Alcoholism, Fort Lauderdale, FL, 19-26 June 2003. We would like to thank Elfar Adalsteinsson, PhD for 
assistance with the three phase analysis, Barton Lane, MD for clinical readings of structural MRI, Anjali Deshmukh, $\mathrm{MD}$ and Anne O'Reilly, PhD for subject clinical evaluation, and Catherine Ju, BA, for subject scheduling and scanning. This research was supported by AA12388, AA05965, AA10723, and AA12999.

\section{REFERENCES}

Agartz I, Brag S, Franck J, Hammarberg A, Okugawa G, Svinhufvud $\mathrm{K}$ et al (2003). MR volumetry during acute alcohol withdrawal and abstinence: a descriptive study. Alcohol Alcoholism 38: 71-78.

Alexander A, Hasan K, Kindlmann G, Parker D, Tsuruda J (2000). A geometric analysis of diffusion tensor measurements of the human brain. Magn Reson Med 44: 283-291.

Alling C, Bostrom K (1980). Demyelination of the mamillary bodies in alcoholism. A combined morphological and biochemical study. Acta Neuropathol (Berl) 50: 77-80.

Badsberg-Jensen G, Pakkenberg B (1993). Do alcoholics drink their neurons away? Lancet 342: 1201-1204.

Basser PJ, Jones DK (2002). Diffusion-tensor MRI: theory, experimental design and data analysis - a technical review. NMR Biomed 15: 456-467.

Bykowski JL, Latour LL, Warach S (2004). More accurate identification of reversible ischemic injury in human stroke by cerebrospinal fluid suppressed diffusion-weighted imaging. Stroke 35: 1100-1106.

de Crespigny A, Moseley M (1998). Eddy current induced image warping in diffusion weighted EPI (abs). Proceedings of the ISMRM, Sydney, NSW, Australia. p 661.

De la Monte SM (1988). Disproportionate atrophy of cerebral white matter in chronic alcoholics. Arch Neurol 45: 990-992.

De Rosa E, Desmond JE, Anderson AK, Pfefferbaum A, Sullivan EV (2004). The human basal forebrain integrates the new and the old. Neuron 41: 825-837.

Estruch R, Nicolas JM, Salamero M, Aragon C, Sacanella E, Fernandez-Sola J et al (1997). Atrophy of the corpus callosum in chronic alcoholism. J Neurol Sci 146: 145-151.

Fein G, Bachman L, Fisher S, Davenport L (1990). Cognitive impairments in abstinent alcoholics. Western J Med 152: 531-537.

Gass A, Birtsch G, Olster M, Schwartz A, Hennerici MG (1998). Marchiafava-Bignami disease: reversibility of neuroimaging abnormality. J Comput Assist Tomogr 22: 503-504.

Harper C, Kril J (1991). If you drink your brain will shrink. Neuropathological considerations. Alcohol Alcoholism 1(Suppl 1): 375-380.

Harper CG, Kril JJ, Daly JM (1987). Are we drinking our neurones away? Br Med J 294: 534-536.

Harper CG, Kril JJ (1993). Neuropathological changes in alcoholics. In: Hunt WA and Nixon SJ (eds). Alcohol Induced Brain Damage: NIAAA Research Monograph No. 22. National Institute of Health: Rockville, MD, USA. pp 39-69.

Hommer D, Momenan R, Rawlings R, Ragan P, Williams W, Rio D et al (1996). Decreased corpus callosum size among alcoholic women. Arch Neurol 53: 359-363.

Hommer DW, Momenan R, Kaiser E, Rawlings RR (2001). Evidence for a gender-related effect of alcoholism on brain volumes. Am J Psychiatry 158: 198-204.

Jernigan TL, Archibald SL, Berhow MT, Sowell ER, Foster DS, Hesselink JR (1991). Cerebral structure on MRI.1. Localization of age-related changes. Biol Psychiatry 29: 55-67.

Kittler J, Illingworth J (1986). Minimum error thresholding. Pattern Recogn 19: 41-47.

Kubicki M, Westin C-F, Maier SE, Mamata H, Frumin M, ErsnerHershfield $\mathrm{H}$ et al (2002). Diffusion tensor imaging and its application to neuropsychiatric disorders. Harvard Rev Psychiatry 10: 324-336.
Lancaster FE (1993). Ethanol and white matter damage in the brain. In: Hunt WA and Nixon SJ (eds). Alcohol-Induced Brain Damage: NIAAA Research Monograph No. 22. National Institute of Health: Rockville, MD, USA. pp 387-399.

Langlais PJ, Zhang SX (1997). Cortical and subcortical white matter damage without Wernicke's encephalopathy after recovery from thiamine deficiency in the rat. Alcohol Clin Exp Res 21: 434-443.

Lewohl J, Wang L, Miles M, Zhang L, Dodd P, Harris R (2000). Gene expression in human alcoholism: microarray analysis of frontal cortex. Alcoholism Clin Exp Res 24: 1873-1882.

Lim KO, Helpern JA (2002). Neuropsychiatric applications of DTI - a review. NMR Biomed 15: 587-593.

Lim KO, Pfefferbaum A (1989). Segmentation of MR brain images into cerebrospinal fluid spaces, white and gray matter. J Comput Assist Tomogr 13: 588-593.

Moseley ME, Cohen Y, Kucharczyk J, Mintorovitch J, Asgari HS, Wendland MF et al (1990). Diffusion-weighted MR imaging of anisotropic water diffusion in cat central nervous system. Radiology 176: 439-445.

Moselhy HF, Georgiou G, Kahn A (2001). Frontal lobe changes in alcoholism: a review of the literature. Alcohol Alcoholism 36: 357-368.

Nelson HE (1982). The National Adult Reading Test (NART). Nelson Publishing Company: Windsor, Canada.

Nixon SJ, Tivis R, Parsons OA (1995). Behavioral dysfunction and cognitive efficiency in male and female alcoholics. Alcoholism Clin Exp Res 19: 577-581.

Nixon SJ (1993). Application of theoretical models to the study of alcohol-induced brain damage. In: Hunt W and Nixon SJ (eds). Alcohol Induced Brain Damage, NIAAA Monograph. National Institute of Health: Rockville, MD, USA. pp 213-228.

Norris DG, Niendorf T, Leibfritz D (1994). Healthy and infarcted brain tissues studied at short diffusion times: the origins of apparent restriction and the reudction in apparent diffusion coefficient. NMR Biomed 7: 304-310.

O’Neill J, Cardenas VA, Meyerhoff DJ (2001). Effects of abstinence on the brain: quantitative magnetic resonance imaging and magnetic resonance spectroscopic imaging in chronic alcohol abuse. Alcoholism Clin Exp Res 25: 1673-1682.

Oscar-Berman M (2000). Neuropsychological vulnerabilities in chronic alcoholism. In: Noronha A, Eckardt $\mathrm{M}$ and Warren $\mathrm{K}$ (eds). Review of NIAAA's Neuroscience and Behavioral Research Portfolio. NIAAA Research Monograph No. 34. Bethesda, MD, USA. pp 437-472.

Otsu N (1979). A threshold selection method from gray-level histograms. IEEE Trans Syst Man Cybernet 9: 63-66.

Paula-Barbosa MM, Tavares MA (1985). Long term alcohol consumption induces microtubular changes in the adult rat cerebellar cortex. Brain Res 339: 195-199.

Pentney RJ (1991). Remodeling of neuronal dendritic networks with aging and alcohol. Alcohol Alcoholism 1(Suppl 1): 393-397.

Pfefferbaum A, Adalsteinsson E, Spielman D, Sullivan EV, Lim KO (1999a). In vivo spectroscopic quantification of the $\mathrm{N}$-acetyl moiety, creatine and choline from large volumes of gray and white matter: effects of normal aging. Magn Reson Med 41: 276-284.

Pfefferbaum A, Lim KO, Desmond J, Sullivan EV (1996). Thinning of the corpus callosum in older alcoholic men: a magnetic resonance imaging study. Alcoholism Clin Exp Res 20: 752-757.

Pfefferbaum A, Lim KO, Zipursky RB, Mathalon DH, Lane B, $\mathrm{Ha} \mathrm{CN}$ et al (1992). Brain gray and white matter volume loss accelerates with aging in chronic alcoholics: a quantitative MRI study. Alcoholism Clin Exp Res 16: 1078-1089.

Pfefferbaum A, Mathalon DH, Sullivan EV, Rawles JM, Zipursky RB, Lim KO (1994). A quantitative magnetic resonance imaging study of changes in brain morphology from infancy to late adulthood. Arch Neurol 51: 874-887. 
Pfefferbaum A, Sullivan EV, Adalsteinsson E, Garrick T, Harper C (2004). Postmortem MR imaging of formalin-fixed human brain. NeuroImage 21: 1585-1595.

Pfefferbaum A, Sullivan EV, Hedehus M, Adalsteinsson E, Lim KO, Moseley M (2000a). In vivo detection and functional correlates of white matter microstructural disruption in chronic alcoholism. Alcoholism Clin Exp Res 24: 1214-1221.

Pfefferbaum A, Sullivan EV, Hedehus M, Lim KO, Adalsteinsson E, Moseley M (2000b). Age-related decline in brain white matter anisotropy measured with spatially corrected echo-planar diffusion tensor imaging. Magn Reson Med 44: 259-268.

Pfefferbaum A, Sullivan EV, Hedehus M, Moseley M, Lim KO (1999b). Brain gray and white matter transverse relaxation time in schizophrenia. Schizophr Res Neuroimaging Sect 91: 93-100.

Pfefferbaum A, Sullivan EV, Mathalon DH, Shear PK, Rosenbloom MJ, Lim KO (1995). Longitudinal changes in magnetic resonance imaging brain volumes in abstinent and relapsed alcoholics. Alcoholism Clin Exp Res 19: 1177-1191.

Pfefferbaum A, Sullivan EV (2003). Increased brain white matter diffusivity in normal adult aging: relationship to anisotropy and partial voluming. Magn Reson Med 49: 953-961.

Pfefferbaum A, Sullivan EV (2002). Microstructural but not macrostructural disruption of white matter in women with chronic alcoholism. Neuroimage 15: 708-718.

Pierpaoli C, Barnett A, Pajevic S, Chen R, Penix L, Virta A et al (2001). Water diffusion changes in Wallerian degeneration and their dependence on white matter architecture. NeuroImage 13: $1174-1185$.

Pratt OE, Rooprai HK, Shaw GK, Thomson AD (1990). The genesis of alcoholic brain tissue injury. Alcohol Alcoholism 25: 217-230.

Putzke J, De Beun R, Schreiber R, De Vry J, Tolle T, Zieglgansberger W et al (1998). Long-term alcohol self-administration and alcohol withdrawal differentially modulate microtubule-associated protein 2 (MAP2) gene expression in the rat brain. Brain Res Mol Brain Res 62: 196-205.

Rickert CH, Karger B, Varchmin-Schultheiss K, Brinkmann B, Paulus W (2001). Neglect-associated fatal Marchiafava-Bignami disease in a non-alcoholic woman. Int J Legal Med 115: 90-93.

Ringer TM, Neumann-Haefelin T, Sobel RA, Moseley ME, Yenari MA (2001). Reversal of early diffusion-weighted magnetic resonance imaging abnormalities does not necessarily reflect tissue salvage in experimental cerebral ischemia. Stroke 32: 2362-2369.

Rosenbloom MJ, Sullivan EV, Pfefferbaum A (2003). Use of MRI to determine brain damage in alcoholics. Alcohol Res Health 27: 146-152.

Rumpel H, Ferrini B, Martin E (1998). Lasting cytotoxic edema as an indicator of irreversible brain damage: a case of neonatal stroke. Am J Neuroradiol 19: 1636-1638.

Sehy JV, Ackerman JJ, Neil JJ (2002). Evidence that both fast and slow water ADC components arise from intracellular space. Magn Reson Med 48: 765-770.

Shear PK, Jernigan TL, Butters N (1994). Volumetric magnetic resonance imaging quantification of longitudinal brain changes in abstinent alcoholics. Alcoholism Clin Exp Res 18: $172-176$.

Silva MD, Omae T, Helme rKG, Li F, Fisher M, Sotak CH (2002). Separating changes in the intra- and extracellular water apparent diffusion coefficient following focal cerebral ischemia in the rat brain. Magn Reson Med 48: 826-837.

Skinner HA, Sheu WJ (1982). Reliability of alcohol use indices: the lifetime drinking history and the MAST. J Studies Alcohol 43: $1157-1170$

Skinner HA (1982). Development and Validation of a Lifetime Alcohol Consumption Assessment Procedure. Addiction Research Foundation: Toronto, Canada.

Sullivan EV, Adalsteinsson E, Hedehus M, Ju C, Moseley M, Lim $\mathrm{KO}$ et al (2001). Equivalent disruption of regional white matter microstructure in aging healthy men and women. Neuroreport 12: 99-104.

Sullivan EV, Pfefferbaum A (2003). Diffusion tensor imaging in normal aging and neuropsychiatric disorders. Eur J Radiol 45: 244-255.

Sullivan EV, Shear PK, Zipursky RB, Sagar HJ, Pfefferbaum A (1994). A deficit profile of executive, memory, and motor functions in schizophrenia. Biol Psychiatry 36: 641-653.

Sullivan EV (2003). Compromised pontocerebellar and cerebellothalamocortical systems: speculations on their contributions to cognitive and motor impairment in nonamnesic alcoholism. Alcoholism Clin Exp Res 27: 1409-1419.

Sullivan EV (2000). Human brain vulnerability to alcoholism: evidence from neuroimaging studies. In: Noronha A, Eckardt $\mathrm{M}$ and Warren K (eds). Review of NIAAA's Neuroscience and Behavioral Research Portfolio, NIAAA Research Monograph No. 34. National Institutes of Health: Bethesda, MD, USA. pp 473-508.

Symonds LL, Archibald SL, Grant I, Zisook S, Jernigan TL (1999). Does an increase in sulcal or ventricular fluid predict where brain tissue is lost? J Neuroimaging 9: 201-209.

Tarnowska-Dziduszko E, Bertrand E, Szpak G (1995). Morphological changes in the corpus callosum in chronic alcoholism. Folia Neuropathol 33: 25-29.

Victor M, Adams RD, Collins GH (1989). The Wernicke-Korsakoff Syndrome and Related Neurologic Disorders Due to Alcoholism and Malnutrition, 2nd ed. F.A. Davis Co.: Philadelphia.

Wechsler D (1987). Wechsler Memory Scale-Revised. The Psychological Corporation: San Antonio, TX.

Westin CF, Maier SE, Mamata H, Nabavi A, Jolesz FA, Kikinis R (2002). Procesing and visualization for diffusion tensor MRI. Med Image Anal 6: 93-108.

Woods R, Grafton S, Holmes C, Cherry S, Mazziotta J (1998). Automated image registration: I. General methods and intrasubject, intramodality validation. J Comput Assist Tomogr 22: 139-152.

Woods RP, Cherry SR, Mazziotta JC (1992). Rapid automated algorithm for aligning and reslicing PET images. J Comput Assist Tomogr 16: 620-633. 